\title{
Molecular clouds in the center of M 81
}

\author{
V. Casasola ${ }^{1,2}$, F. Combes ${ }^{2}$, D. Bettoni ${ }^{3}$, and G. Galletta ${ }^{1}$ \\ 1 Dipartimento di Astronomia, Università di Padova, Vicolo dell' Osservatorio 2, 35122 Padova, Italy \\ e-mail: [viviana.casasola;giuseppe.galletta]@unipd.it \\ ${ }^{2}$ LERMA, Observatoire de Paris, 61 avenue de l'Observatoire, 75014 Paris, France \\ e-mail: francoise.combes@obspm.fr \\ 3 INAF, Osservatorio Astronomico di Padova, Vicolo dell'Osservatorio 5, 35122 Padova, Italy \\ e-mail: daniela.bettoni@oapd.inaf.it
}

Received 14 May 2007 / Accepted 18 July 2007

\section{ABSTRACT}

\begin{abstract}
We investigate the molecular gas content and the excitation and fragmentation properties in the central region of the spiral galaxy Messier 81 in both the ${ }^{12} \mathrm{CO}(1-0)$ and ${ }^{12} \mathrm{CO}(2-1)$ transitions. We have recently observed the two transitions of $\mathrm{CO}$ in the $\mathrm{M} 81$ center with A, B, and HERA receivers of the IRAM 30-m telescope. We find no CO emission in the inner $\sim 300 \mathrm{pc}$ and a weak molecular gas clump structure at a distance of around $460 \mathrm{pc}$ from the nucleus. Observations of the first two CO transitions allowed us to compute the line ratio, and the average $I_{21} / I_{10}$ ratio is 0.68 for the M 81 center. This low value, atypical both of the galactic nuclei of spiral galaxies and of interacting systems, is probably associated to diffuse gas with molecular hydrogen density that is not high enough to excite the $\mathrm{CO}$ molecules. After analyzing the clumping properties of the molecular gas in detail, we identify very massive giant molecular associations (GMAs) in $\mathrm{CO}(2-1)$ emission with masses of $\sim 10^{5} M_{\odot}$ and diameters of $\sim 250 \mathrm{pc}$. The deduced $N\left(\mathrm{H}_{2}\right) / I_{\mathrm{CO}}$ ratio for the individually resolved GMAs, assumed to be virialized, is a factor of $\sim 15$ higher than the standard Galactic value, showing - as suspected - that the $\mathrm{X}$ ratio departs significantly from the mean for galaxies with an unusual physics of the molecular gas.
\end{abstract}

Key words. galaxies: ISM - galaxies: spiral - ISM: clouds - ISM: molecules

\section{Introduction}

The molecular gas content of Messier 81, one of the nearest face-on spirals (SA(s)ab; de Vaucouleurs et al. 1991), has always been a puzzle as seen in the first observations by Solomon \& de Zafra (1975) and Combes et al. (1977). The CO emission appears very weak in this galaxy, especially in the central regions, and the molecular content seems to be confined to the HII regions in the spiral arms, between 4 and $7 \mathrm{kpc}$ from the center (Solomon et al. 1987; Brouillet et al. 1988, 1991; Sage \& Westpfahl 1991; Sakamoto et al. 2001). Two studies (Solomon \& de Zafra 1975; Combes et al. 1977) searched the molecular gas in the center of M 81 but only found upper limits or failed to detect any - probably due to the low CO emission - so they did not draw any conclusions from this result. The first detection of the molecular gas in the center of M 81 was by Sage \& Westpfahl (1991), who found that the peak intensities are a factor 4 lower than those observed by Brouillet et al. (1988) in the outer disk. After the weak detection by Sage \& Westpfahl (1991), only Sakamoto et al. (2001) have studied the CO emission in the center of M 81 and also found a particular molecular gas physics. M 81 has also been studied in all components of its interstellar medium (ISM), from radio (e.g. Beck et al. 1985; Bietenholz et al. 1996), through optical and UV (e.g. Ho et al. 1996), to X-ray bands (e.g. Pellegrini et al. 2000).

In addition to the weak $\mathrm{CO}$ emission, the $\mathrm{HI}$ profile also reflects a central deficiency in atomic gas. The HI gas is abundant only in the inner spiral arms of the galaxy (Visser 1980; Allen et al. 1997), and the shapes and the motions observed in HI in the spiral arms quite clearly obey the predictions of the densitywave theory. This strong density wave is attributed to the tidal interaction with the other galaxies of the M 81 group, especially M 82 and NGC 3077 (Kaufman et al. 1989).

In general, interacting galaxies like $\mathrm{M} 81$ possess higher $\mathrm{CO}$ luminosity by almost an order of magnitude than non-interacting galaxies (Braine \& Combes 1993; Combes et al. 1994; Casasola et al. 2004). Then, M 81 seems an exception remarkably poor for its $\mathrm{CO}$ emission. For this reason, M 81 is a kind of prototype of CO-poor galaxies, as is Andromeda or the center of NGC 7331, a lesser extent.

M 81 is also a good candidate for exploring the problem of the varying $\mathrm{CO}$ to $\mathrm{H}_{2}$ conversion ratio $\left(X=N\left(\mathrm{H}_{2}\right) / I_{\mathrm{CO}}\right)$. This ratio is well-known for varying substantially in dwarf galaxies, for which the metallicity is deficient (Rubio et al. 1993; Taylor et al. 1998), while for galaxies like M 81 that are giants with normal metallicity, the problem of the low $\mathrm{CO}$ emission has not been yet explored much. The $\mathrm{CO}$ to $\mathrm{H}_{2}$ conversion ratio for M 81 is suspected - on the base of the observations that are already available - of departing significantly from the mean $\left(X=2.3 \times 10^{20} \mathrm{~mol} \mathrm{~cm}^{-2}\left(\mathrm{~K} \mathrm{~km} \mathrm{~s}^{-1}\right)^{-1}\right.$, Strong et al. 1988), because galaxies with low CO content, such as M 31, NGC 55, or LMC, show a much larger $X$ factor (Nakai \& Kuno 1995).

In this paper, we assume M 81 galaxy at a distance of $3.6 \mathrm{Mpc}$ and adopt $H_{0}=70 \mathrm{~km} \mathrm{~s}^{-1} \mathrm{Mpc}^{-1}$. We present the $\mathrm{CO}(1-0)$ and $\mathrm{CO}(2-1)$ observations of the central $\sim 1.5 \mathrm{kpc}$ of M 81 carried out at the IRAM 30-m telescope. The primary goal of this work is to investigate the amount and distribution of the molecular gas component in the central regions.

The structure of the paper is as follows. In Sect. 2 the observations performed and the techniques of the data reduction used are described. In Sect. 3 we present the observational results, and in particular, the $R_{21}=I_{21} / I_{21}$ line ratio is studied and analyzed 
for all the offsets observed that give information on the physical properties of the gas in the M 81 center. The clumpy nature of the molecular gas, the virial equilibrium, and the X-ratio of the resolved individual clouds are discussed in Sect. 4. Our results are compared with previous studies of the molecular gas in M 81 and discussed, giving their physical interpretation in Sect. 5. Finally, in Sect. 6 we summarize the main results.

\section{Observations and data reduction}

The observations were made from 2 from 6 January 2006 and from 31 March to 1 April 2006 at the IRAM 30-m telescope at Pico Veleta, near Granada (Spain). We used two different configurations for the two observing runs and we covered the central $90^{\prime \prime} \times 90^{\prime \prime}$, where at the distance of M $81,1^{\prime \prime}=17.4 \mathrm{pc}$. The receiver-cabin optic system of the IRAM 30-m telescope is optimized to observe with up to four different receivers simultaneously (Wild 1999).

In the first observing run we used the A100, A230, B100, and B230 heterodyne receivers of the telescope, which simultaneously observed at both $115 \mathrm{GHz}$ (A100 and B100, at $\lambda \simeq$ $2.6 \mathrm{~mm}$, where $\lambda$ is the wavelength) and $230 \mathrm{GHz}$ (A230 and B230, at $\lambda \simeq 1.3 \mathrm{~mm}$ ). We used the $1 \mathrm{MHz}$ back-ends with an effective total bandwidth of $512 \mathrm{MHz}$ at $2.6 \mathrm{~mm}$ and an autocorrelator at $1.3 \mathrm{~mm}$. We also used the $4 \mathrm{MHz}$ filterbanks with an effective total bandwidth of $1024 \mathrm{MHz}$ at $1.3 \mathrm{~mm}$. These arrangements provided a velocity coverage of $1300 \mathrm{~km} \mathrm{~s}^{-1}$ in both the $\mathrm{CO}(1-0)$ and $\mathrm{CO}(2-1)$ lines, and all the measurements were performed in "wobbler-switching" mode. This observing mode has a minimum phase time for spectral line observations of $2 \mathrm{~s}$ and a maximum beam throw of 240 ". The advantage of the "wobblerswitching" mode is to give very flat baselines without any ripple in most cases. The half power beam widths (HPBW) are $22^{\prime \prime}$ and $12^{\prime \prime}$ for the $\mathrm{CO}(1-0)$ and $\mathrm{CO}(2-1)$ lines, respectively, and the typical system temperatures were $\sim 100 \mathrm{~K}$ at $115 \mathrm{GHz}$ and $\sim 400 \mathrm{~K}$ at $230 \mathrm{GHz}$. The positions observed with A and B receivers were 81 for both lines.

In the second observing run, we used the Heterodyne Receiver Array HERA (Schuster et al. 2004), a focal array of 18 SIS receivers, 9 for each polarization, tuned to the $\operatorname{CO}(2-1)$ line for M 81. The 9 pixels are arranged in the form of a centerfilled square and are separated by $24^{\prime \prime}$. The sampling was $6^{\prime \prime}$, and a homogeneous mapping procedure was used to regularly sweep a $12 \times 12$ pixel map, filling the intrinsic square of $66^{\prime \prime} \times 66^{\prime \prime}$. The typical system temperature was $\sim 400 \mathrm{~K}$. Also in this case we used the "wobbler-switching" observing mode, and the pointing accuracy was $\sim 3$ " rms. The WILMA backend was used, providing a $1 \mathrm{GHz}$ bandwidth for each of the 18 receivers. The bands contain $2 \times 465 \mathrm{MHz}(=930 \mathrm{MHz})$ channels spaced by $2 \mathrm{MHz}$ of resolution. The total bandwidth corresponds to $1300 \mathrm{~km} \mathrm{~s}^{-1}$ with a velocity resolution of $2.6 \mathrm{~km} \mathrm{~s}^{-1}$. The map realized with HERA included 144 positions.

The line intensity scale throughout this paper is in units of $T_{\mathrm{mb}}$, the beam-average radiation temperature. The value of $T_{\mathrm{mb}}$ is related to $T_{\mathrm{A}}^{*}$, the equivalent antenna temperature - corrected for rear spillover and ohmic losses - reported above the atmosphere, by $\eta=T_{\mathrm{A}}^{*} / T_{\mathrm{mb}}$, where $\eta$ is the telescope main-beam efficiency, $\eta$ defined as the ratio between the beam efficiency $B_{\text {eff }}$ and the forward efficiency $F_{\text {eff }}, \eta=B_{\text {eff }} / F_{\text {eff }}$. The beam efficiency is usually derived from continuum measurements of planets, while the forward efficiency is obtained by pointing the antenna at different elevations and measuring the $T_{\mathrm{A}}^{*}$ of the sky at each step. The sky temperatures are successively fitted by an exponential function of the air mass, and the forward efficiency
Table 1. Fundamental parameters for M 81 (at a distance of $3.6 \mathrm{Mpc}$ adopted for $\mathrm{M} 81,1^{\prime \prime}$ is $\sim 17.4 \mathrm{pc}$ ).

\begin{tabular}{lcccc}
\hline \hline $\begin{array}{l}\text { RA } \\
(\mathrm{J} 2000.0)\end{array}$ & $\begin{array}{c}\text { Dec } \\
(\mathrm{J} 2000.0)\end{array}$ & $\begin{array}{c}V \\
\left(\mathrm{~km}^{-1} \mathrm{~s}\right)\end{array}$ & $\begin{array}{c}v \\
\mathrm{GHz}\end{array}$ & Line \\
\hline $09: 55: 33$ & $69: 03: 55$ & -34 & 115.271 & ${ }^{12} \mathrm{CO}(1-0)$ \\
& & & 230.538 & ${ }^{12} \mathrm{CO}(2-1)$ \\
\hline
\end{tabular}

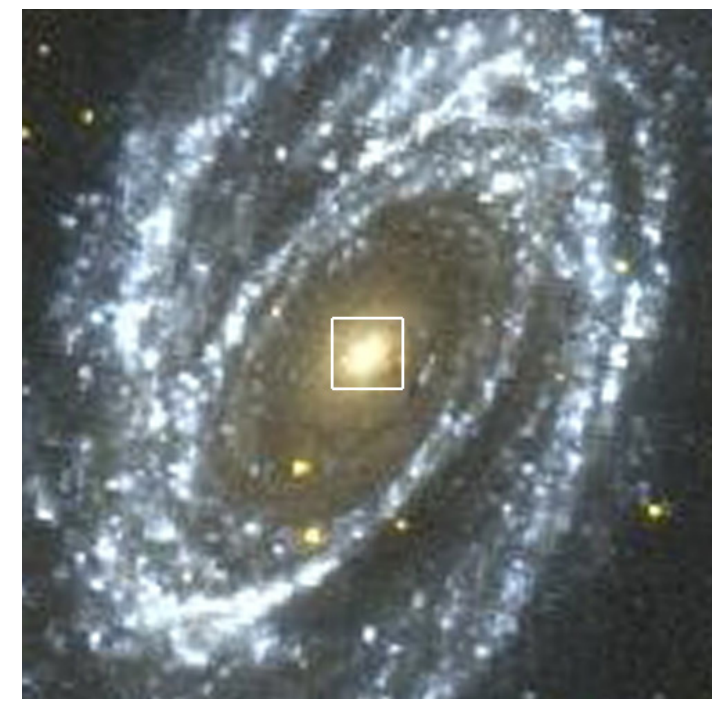

Fig. 1. The field of our observations (white box, 40 arcsec in size) superposed on a GALEX composite image of M 81. In the figure, FUV is in the blue channel and NUV the yellow one.

is derived with the atmospheric opacity. At $115 \mathrm{GHz} \eta=0.79$, while $\eta_{B_{\text {eff }}}=0.57$ at $230 \mathrm{GHz}$.

The data were reduced with the GILDAS ${ }^{1}$ software package. Some spectra with random, highly non-linear baselines were suppressed, and linear baselines were subtracted from all the remaining spectra. In Table 1 the fundamental parameters for the M 81 observations are summarized.

\section{Molecular gas emission}

The region observed with the A and B receivers of the IRAM 30$\mathrm{m}$ telescope in the two CO lines covers the central $\sim 1.6 \mathrm{kpc}$ of M 81. The size and the location of the region covered by our $\mathrm{CO}$ observations are shown in Fig. 1 as a square superposed on a GALEX image of the galaxy. The $\mathrm{CO}$ emission, both in $\mathrm{CO}(1-0)$ and $\mathrm{CO}(2-1)$ lines, comes from different regions of the galaxy nucleus. In agreement with Sage \& Westpfahl (1991) and Sakamoto et al. (2001), we find that the nucleus presents some regions devoid of molecular gas, but also that there are other regions in which the $\mathrm{CO}$ is clearly detected and the signal is strong (Fig. 2).

In particular, the central $\sim 300 \mathrm{pc}$ region is devoid of $\mathrm{CO}(1-0)$ emission in agreement, for instance, with Brouillet et al. (1988) and Sakamoto et al. (2001), but there is a main molecular gas clump structure at a distance of around $460 \mathrm{pc}$ from the nucleus in the northeast direction (Fig. 3), which is very similar to the maximum intensity detected by Sakamoto et al. (2001). In addition to the $\mathrm{CO}$ arc in the northeast direction, we also detected some emission to the southwest one.

${ }^{1}$ http://www.iram.fr/IRAMFR/GILDAS/ 

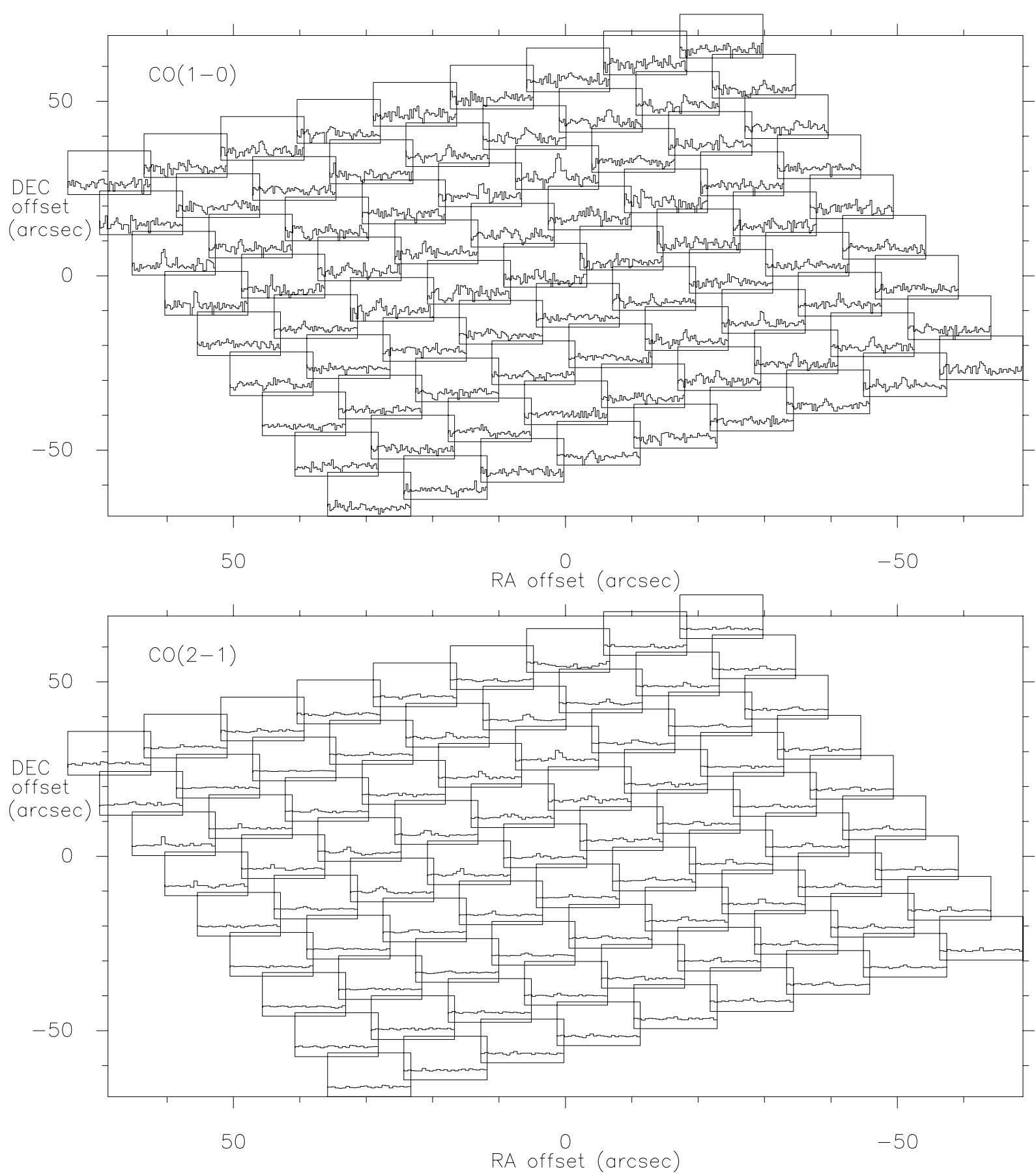

Fig. 2. Map of the observed M 81 central region. Each spectrum has a velocity scale from -600 to $600 \mathrm{~km} \mathrm{~s}^{-1}$, and a temperature scale from -20 to $70 \mathrm{mK}$. The positions are offsets relative to the M 81 nucleus assumed of coordinates RA $\mathrm{J}_{2000.0}=09^{\mathrm{h}} 55^{\mathrm{m}} 33^{\mathrm{s}}, \mathrm{Dec}_{\mathrm{J} 2000.0}=69^{\circ} 03^{\prime} 55^{\prime \prime}$. Upper panel: observations of the ${ }^{12} \mathrm{CO}(1-0)$ line. Bottom panel: observations of the ${ }^{12} \mathrm{CO}(2-1)$ line convolved to the ${ }^{12} \mathrm{CO}(1-0)$ beam resolution $\left(22^{\prime \prime}\right)$.

\subsection{HERA-receiver results}

The observations done with the HERA receiver cover the central $\sim 1.3 \mathrm{kpc}$, similar at the central $\sim 1.6 \mathrm{kpc}$ reported above observed with the A and B receivers. These observations confirm that the molecular gas emission in the M 81 center is at a low temperature. Figure 4 shows the spectral map and Fig. 5 the integrated contours obtained with our observations. HERA observations performed for the ${ }^{12} \mathrm{CO}(2-1)$ line also reveal "islands" of molecular gas in the nuclear region. There are regions completely devoid of emission, but also central offsets with a faint but detectable emission. The pointings with signals $\geq 2 \sigma$ have peak temperatures between $16 \mathrm{mK}$ and $65 \mathrm{mK}$. There is good correspondence between the emission in $\mathrm{CO}(2-1)$ found with HERA receiver and with A and B receivers, as shown in Fig. 6 where the distributions found with the two observing configurations overlap.

\subsection{The $C O(2-1)$ to $C O(1-0)$ line ratio}

The combination of observations of the $\mathrm{CO}(1-0)$ and $\mathrm{CO}(2-1)$ lines allow us to compute the line ratio and to study the physical properties of the gas more in detail, such as excitation temperature and optical depth. 


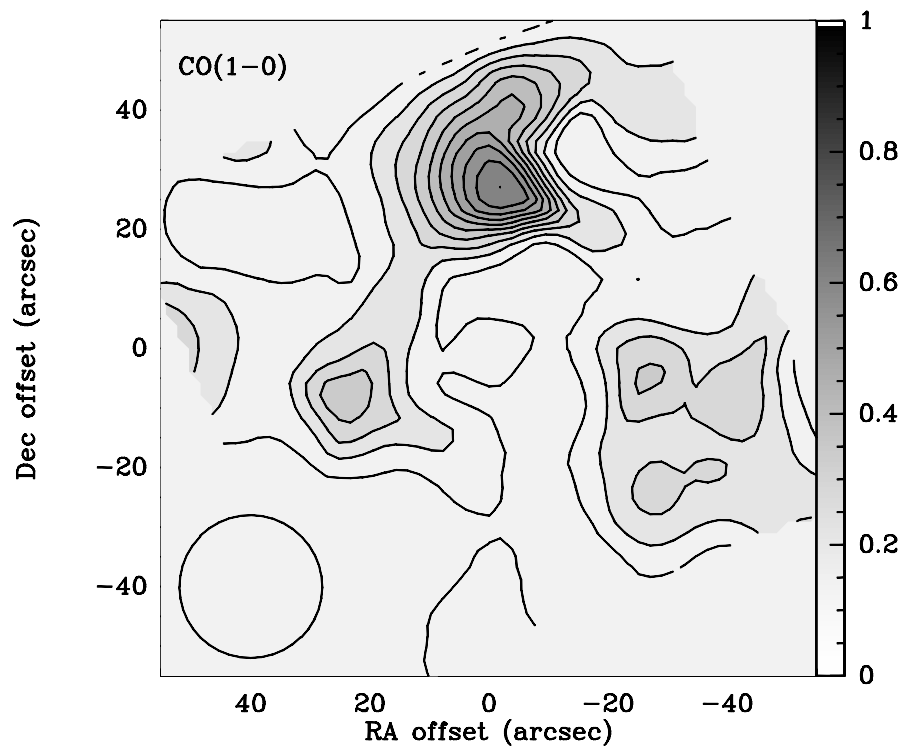

Fig. 3. Integrated ${ }^{12} \mathrm{CO}(1-0)$ emission. The contour levels are between 0.701 to $7.01 \mathrm{~K} \mathrm{~km} \mathrm{~s}^{-1}$ in steps of $0.701 \mathrm{~K} \mathrm{~km} \mathrm{~s}^{-1}$. The beam of $22^{\prime \prime}$ is indicated at the bottom left.

The interpretation of the $R_{21}=I_{21} / I_{21}=\int T_{\mathrm{mb}}(2-1) \mathrm{d} v /$ $\int T_{\mathrm{mb}}(1-0) \mathrm{d} v$ line ratio is quite complex and usually simplified with standard assumptions. The main assumption is to separate the analysis of the $R_{21}$ ratio into two limiting cases: the optically thin case and the optically thick case. The analysis of these two limiting cases for the $\mathrm{CO}$ molecule has shown two important things. First, for high temperatures, the $R_{21}$ ratio asymptotically approaches a value of 4 in the optically thin case, while it only approaches 1 in the optically thick case. If 1 is the limit for an optically thick gas, $R_{21}$ ratios $>1$ are signatures of optically thin CO emission. Second, in the optically thin limit, the $R_{21}$ ratio does not drop below 1 until the excitation temperature drops below $8 \mathrm{~K}$. This situation is improbable, but not impossible, especially if the excitation of the gas is subthermal.

The ${ }^{12} \mathrm{CO}$ lines are optically thick in most galaxies, including the Milky Way, but there are other galaxies where the problem is still open, like M 82 - the interacting companion of M 81 (Loiseau et al. 1990) or NGC 3628 (Reuter et al. 1991). When studying the line ratio for a large sample (118) of nearby spiral galaxies, Braine \& Combes (1992) saw that subthermal excitation or different gas filling factors are only required for explaining line ratios less than 0.7 .

With line ratios of $0.7,0.8$, and 0.9 , the excitation temperatures are expected to be $7 \mathrm{~K}, 10.5 \mathrm{~K}$, and $21 \mathrm{~K}$, respectively. These low line ratios can be explained considering that at high gas densities in the heating mechanism the dust-gas collisions are more important than cosmic rays that ionize $\mathrm{H}_{2}$ or via formation of $\mathrm{H}_{2}$ on dust grains. When neglecting the heating from gravitational collapse or magnetic sources and assuming the gas in local thermal equilibrium $\left(T_{\mathrm{ex}}=T_{\mathrm{kin}}\right)$, Braine \& Combes (1992) derived a relationship between gas temperature and dust temperature at high densities. At gas densities of $10^{4} \mathrm{~cm}^{-3}$, which is required to approximately thermalize the $J=2$ level of $\mathrm{CO}$, and dust temperatures of $30 \mathrm{~K}$, the equilibrium gas temperature is $10-13 \mathrm{~K}$. Considering that in the clouds the dust is probably warmer in the vicinity of OB stars and lower elsewhere, the gas heated by the warm dust at $50-60 \mathrm{~K}$ will reach the thermal equilibrium at temperatures of $15-20 \mathrm{~K}$. The $J=2$ level of CO is populated at these temperatures, and Braine \& Combes (1992) computed that the expected line ratios are between 0.7 and 0.9 . In addition, it is important to remember that the $\mathrm{CO}$ emission is not always thermalized and that the excitation temperature may change along the line of sight and within a single beam.

We computed the $R_{21}$ integrated intensity ratios for the offsets observed in the M 81 center, after smoothing the ${ }^{12} \mathrm{CO}(2-1)$ data to the ${ }^{12} \mathrm{CO}(1-0)$ beam resolution $\left(22^{\prime \prime}\right)$ and correcting for the different beam efficiencies. In Table 2 the main results of the Gaussian fits of the observations are listed. In this table, Cols. (1) and (2) are the offsets $(\Delta \alpha$ and $\Delta \beta)$ referred to the center of the galaxy, which coordinates are given in Table 1; Cols. (3) and (6) are the beam-average radiation temperatures $\left(T_{\mathrm{mb}}\right)$ for the two transitions; Cols. (4) and (7) are the line-widths $(\Delta v)$ for the two transitions; Col. (9) is the $R_{21}=I_{21} / I_{10}$ convolved line ratios; Cols. (5) and (8) are the CO intensities of the two transitions obtained from Gaussian fits.

The first striking note is the low temperature both in $\mathrm{CO}(1-$ $0)$ and $\mathrm{CO}(2-1)$ transitions, which characterizes the emission of all observed positions in the M 81 central region. For those detections $\geq 2 \sigma$, the average brightness temperature in $\mathrm{CO}(1-0)$ is $33.44 \mathrm{mK}$, while in $\mathrm{CO}(2-1)$ it is $21.47 \mathrm{mK}$.

The $R_{21}$ ratios found in the central regions of M 81 are quite low: where the $\mathrm{CO}(1-0)$ emission is particularly weak, that of the $\mathrm{CO}(2-1)$ line appears weaker or difficult to detect, producing a low $R_{21}$ value. The average $R_{21}$ ratio for the center of M 81 is 0.68 , a value atypical of galactic nuclei. Actually, in CO surveys (Braine \& Combes 1992) the central regions (inner $\mathrm{kpc}$ ) of the galaxies have, on average, $R_{21} \sim 0.89$, rarely reaching higher ratios (e.g. for NGC 3310). According to Braine \& Combes (1992), this happens because the inner molecular gas is optically thick in ${ }^{12} \mathrm{CO}$ lines and is cool but not cold $(T>10 \mathrm{~K})$. Our low ratio for M 81 is lower than the average value of 0.89 for galaxies similar in morphology and distance. In addition, isolated galaxies appear to have line ratios that are lower than interacting/perturbed galaxies (Braine \& Combes 1992). M 81 exhibits a low value, despite being a classical example of interaction.

Studies of the disk in single galaxies show, in general, lower line ratios than those in the galactic nuclei. IC 342 (Eckart et al. 1990), NGC 6946 (Casoli et al. 1990), and M 51 (Garcia-Burillo et al. 1993) are examples in which the $R_{21}$ ratio typically decreases from $\sim 1$ in the galactic nuclei to $0.4-0.6$ in the spiral arms.

To test if $R_{21}$ in $\mathrm{M} 81$ shows a similar trend and the ratio decreases is necessary to use data in the literature. There is only one observational study on M 81 with data of the spiral arms in both $\mathrm{CO}(1-0)$ and $\mathrm{CO}(2-1)$ lines (Brouillet et al. 1991). We used Fig. 2 of Brouillet et al. (1991) to study only the fields with detections for the two lines and computed the corresponding $R_{21}$ ratios after the transformation from detected $T_{\mathrm{A}}^{*}$ to $T_{\mathrm{mb}}$, needed for the comparison with our data. The results of this analysis are collected in Table 3. In this table, Cols. (1) and (2) are the fields and the corresponding coordinates respectively defined in Fig. 2 of Brouillet et al. (1991); Cols. (3) and (4) are the intensities lines $I_{10}$ and $I_{21}$ respectively computed from the $T_{\mathrm{A}}^{*}$ detected by Brouillet et al. (1991); Col. (5) is the $R_{21}=I_{21} / I_{10}$ line ratio. Table 3 shows that the $R_{21}$ line ratio spans in a wide range of values, without a clear radial trend. For instance, the regions N5 and N7, that are in the same area of the northeastern spiral arm, show very different values for $R_{21}$. This confirms the "anomalous" $R_{21}$ behavior of M 81 with respect to other spiral galaxies.

In conclusion, M 81 is a galaxy with low intensity and an anomalous behavior of CO lines. In this respect, it is similar 


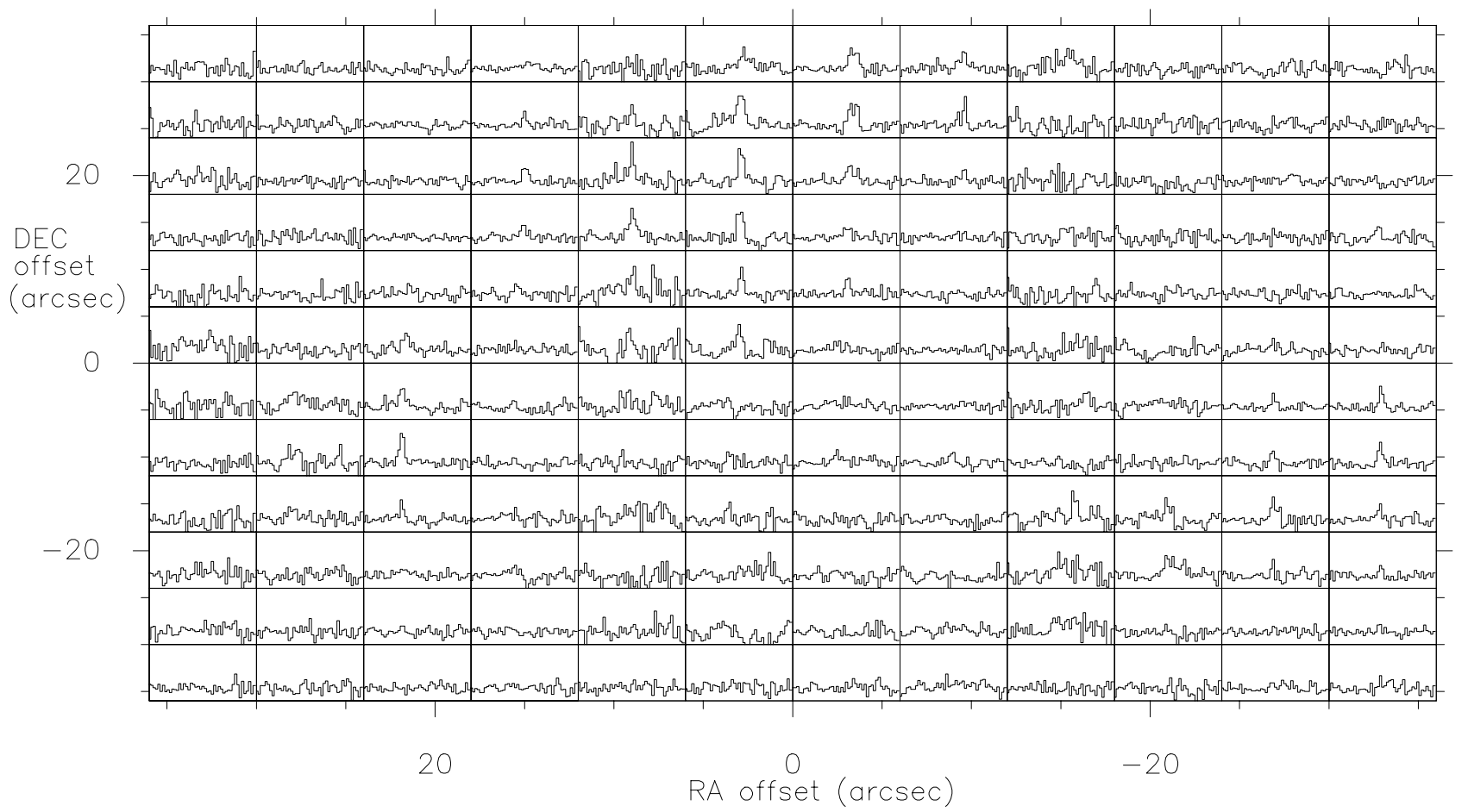

Fig. 4. ${ }^{12} \mathrm{CO}(2-1)$ map of the M 81 central region observed with the HERA receiver. Each spectrum has a velocity scale from -600 to $600 \mathrm{~km} \mathrm{~s}{ }^{-1}$ and a temperature scale from -20 to $70 \mathrm{mK}$. The positions are offsets relative to the $\mathrm{M} 81$ nucleus assumed of coordinates $\mathrm{RA}_{\mathrm{J} 2000.0}=09^{\mathrm{h}} 55^{\mathrm{m}} 33^{\mathrm{s}}$, $\operatorname{Dec}_{\mathrm{J} 2000.0}=69^{\circ} 03^{\prime} 55^{\prime \prime}$.

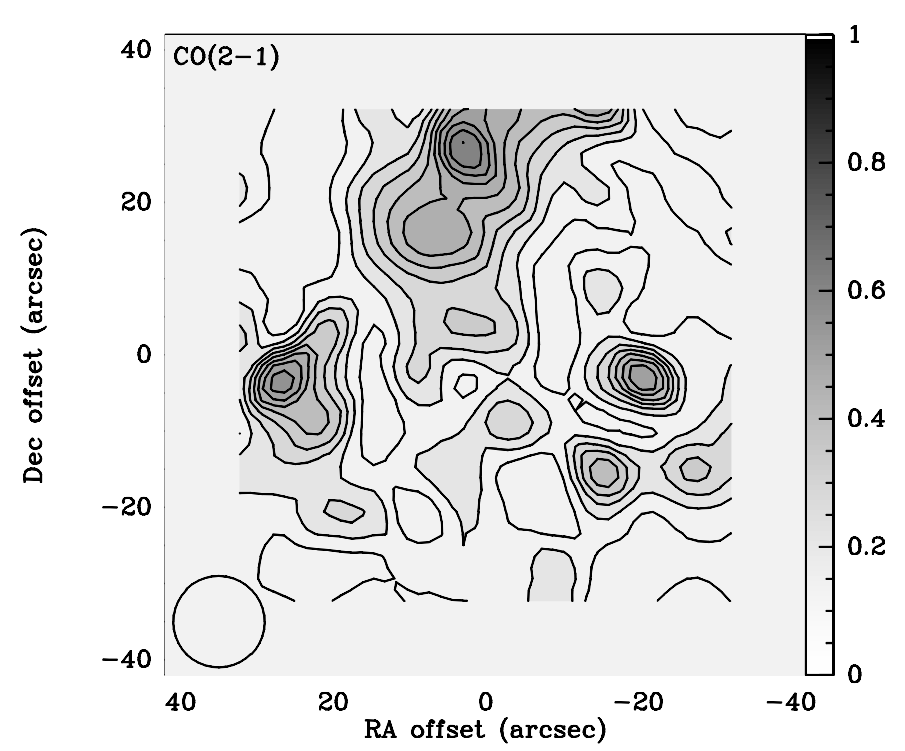

Fig. 5. Integrated ${ }^{12} \mathrm{CO}(2-1)$ emission. The contour levels are between 0.410 to $4.10 \mathrm{~K} \mathrm{~km} \mathrm{~s}^{-1}$ in steps of $0.410 \mathrm{~K} \mathrm{~km} \mathrm{~s}^{-1}$. The beam of $12^{\prime \prime}$ is indicated at the bottom left.

to the central CO-poor galaxies NGC 7331 (Young \& Scoville 1982; Young et al. 1995), which has $R_{21} \sim 0.54$ (Israel \& Baas 1999) and M 31, where similar low ratios have been found for individual dark clouds in the central region (Allen \& Lequeux 1993). In this case, the authors attributed this fact to a subthermal excitation of the gas, at least for the $\mathrm{CO}(2-1)$ transition. These low ratios may be understood if coming from cool or cold, diffuse gas with a molecular hydrogen density $\left(n\left(\mathrm{H}_{2}\right)\right)$ not high enough to excite the $\mathrm{CO}$ molecules.

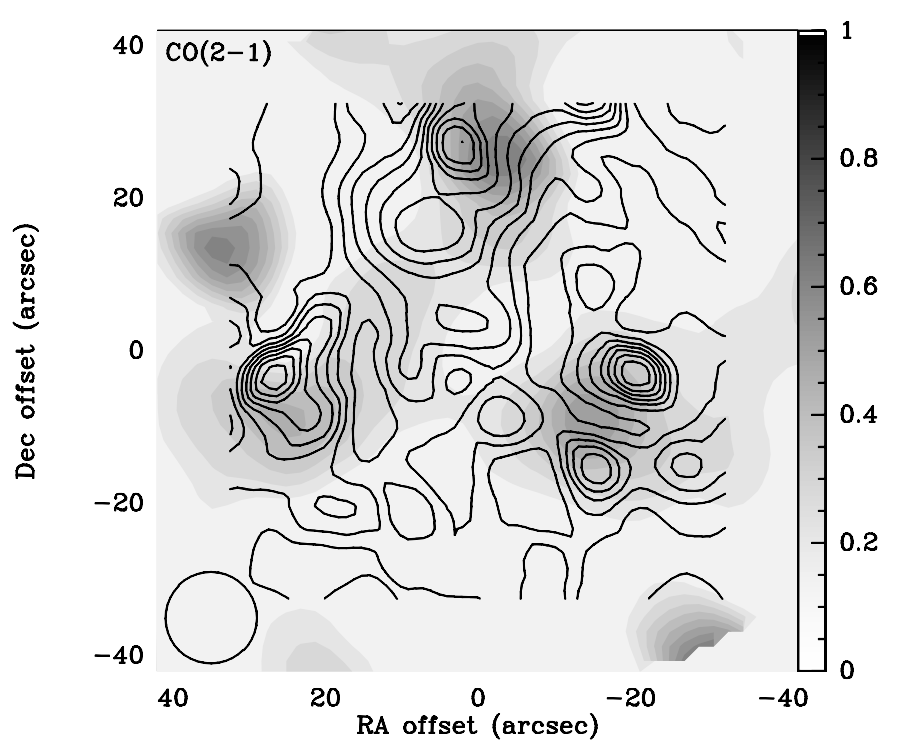

Fig. 6. Integrated ${ }^{12} \mathrm{CO}(2-1)$ emission detected with the HERA receiver superposed on the ${ }^{12} \mathrm{CO}(2-1)$ emission found with $\mathrm{A}$ and $\mathrm{B}$ receivers. The contour levels are the same as shown in Fig. 5 between 0.410 to $4.10 \mathrm{~K} \mathrm{~km} \mathrm{~s}^{-1}$ in steps of $0.410 \mathrm{~K} \mathrm{~km} \mathrm{~s}^{-1}$ and the intensity levels detected with A and B receivers are in grey scale. The beam of $12^{\prime \prime}$ is indicated at the bottom left. The intensity peaks detected with the two observing configurations agree quite well between them.

\section{Clumping properties of the gas}

There is evidence that molecular clouds have a high degree of internal fragmentation on all scales: this medium is defined as clumpy. Regan et al. (2001) and Helfer et al. (2003), used the BIMA SONG survey - a systematic imaging survey in $\mathrm{CO}(1-0)$ 
Table 2. $\mathrm{CO}(J=1-0)$ and $\mathrm{CO}(J=2-1)$ intensity lines where $\mathrm{CO}$ emission is detected (detection level $\geq 2 \sigma$ ).

\begin{tabular}{|c|c|c|c|c|c|c|c|c|}
\hline $\begin{array}{r}\text { Offset } \\
\Delta \alpha\left({ }^{\prime \prime}\right) \\
\text { (1) }\end{array}$ & $\begin{array}{r}\text { Offset } \\
\Delta \beta\left({ }^{\prime \prime}\right) \\
\text { (2) }\end{array}$ & $\begin{array}{c}T_{\mathrm{mb}}(1-0) \\
{[\mathrm{mK}]} \\
(3)\end{array}$ & $\begin{array}{c}\Delta v(1-0) \\
{\left[\mathrm{km} \mathrm{s}^{-1}\right]} \\
(4)\end{array}$ & $\begin{array}{c}T_{\mathrm{mb}}(1-0) \mathrm{d} v \\
{\left[\mathrm{~K} \mathrm{~km} \mathrm{~s}^{-1}\right]} \\
(5)\end{array}$ & $\begin{array}{c}T_{\mathrm{mb}}(2-1) \\
{[\mathrm{mK}]} \\
(6)\end{array}$ & $\begin{array}{c}\Delta v(2-1) \\
{\left[\mathrm{km} \mathrm{s}^{-1}\right]} \\
(7)\end{array}$ & $\begin{array}{c}\int T_{\mathrm{mb}}(2-1) \mathrm{d} v \\
{\left[\mathrm{~K} \mathrm{~km} \mathrm{~s}^{-1}\right]} \\
(8)\end{array}$ & $R_{21}$ \\
\hline-51.9 & -30.1 & 29.04 & 74.01 & 2.15 & 10.23 & 83.21 & 0.85 & 0.40 \\
\hline-47.2 & -19.1 & 30.16 & 59.01 & 1.78 & 12.73 & 82.55 & 1.05 & 0.59 \\
\hline-42.5 & -8.0 & 28.34 & 88.23 & 2.50 & 14.55 & 115.60 & 1.68 & 0.67 \\
\hline-37.8 & 3.0 & 16.97 & 79.81 & 1.35 & & & & \\
\hline-36.2 & -23.8 & 34.22 & 59.89 & 2.05 & 16.67 & 87.36 & 1.46 & 0.71 \\
\hline-31.5 & -12.7 & 38.18 & 52.72 & 2.01 & 23.87 & 73.12 & 1.75 & 0.87 \\
\hline-26.8 & -1.7 & 18.09 & 150.30 & 2.72 & 20.39 & 97.33 & 1.98 & 0.73 \\
\hline-25.1 & -28.4 & 31.67 & 61.07 & 1.93 & 25.13 & 52.02 & 1.31 & 0.68 \\
\hline-20.4 & -17.4 & 33.16 & 47.82 & 1.59 & & & & \\
\hline-19.1 & 47.2 & 31.85 & 56.16 & 1.79 & & & & \\
\hline-8.0 & 42.5 & 38.36 & 96.15 & 3.69 & 16.16 & 70.65 & 1.14 & 0.31 \\
\hline-1.7 & 26.8 & 68.42 & 102.50 & 7.01 & 40.16 & 102.10 & 4.10 & 0.58 \\
\hline 3.0 & 37.8 & 27.99 & 152.80 & 4.28 & 24.86 & 116.50 & 2.90 & 0.68 \\
\hline 6.4 & -15.7 & 12.52 & 113.40 & 1.42 & & & & \\
\hline 9.4 & 22.1 & 39.60 & 78.91 & 3.12 & & & & \\
\hline 14.1 & 33.1 & 34.47 & 66.19 & 2.28 & 22.30 & 80.29 & 1.79 & 0.78 \\
\hline 15.7 & 6.4 & 41.16 & 44.16 & 1.82 & 18.33 & 94.03 & 1.72 & 0.95 \\
\hline 22.1 & -9.4 & 31.75 & 96.58 & 3.07 & 20.61 & 121.10 & 2.50 & 0.81 \\
\hline 26.8 & 1.7 & 21.40 & 67.65 & 1.45 & 22.95 & 58.55 & 1.34 & 0.93 \\
\hline 33.1 & -14.1 & 21.76 & 43.95 & 0.96 & & & & \\
\hline 48.9 & -7.7 & 58.60 & 32.25 & 1.89 & 19.20 & 52.02 & 1.00 & 0.53 \\
\hline 53.6 & 3.3 & 47.86 & 55.29 & 2.65 & 35.44 & 61.72 & 2.19 & 0.83 \\
\hline
\end{tabular}

Table 3. $R_{21}$ line ratio for spiral arms of M 81 by Brouillet et al. (1991).

\begin{tabular}{lrccc}
\hline \hline Field & $\begin{array}{r}\text { Offsets } \\
\left({ }^{\prime \prime},{ }^{\prime}\right)\end{array}$ & $\begin{array}{c}\int T_{\mathrm{mb}}(1-0) \mathrm{d} v \\
{\left[\mathrm{~K} \mathrm{~km} \mathrm{~s}^{-1}\right]}\end{array}$ & $\begin{array}{c}\int T_{\mathrm{mb}}(2-1) \mathrm{d} v \\
{\left[\mathrm{~K} \mathrm{~km} \mathrm{~s}^{-1}\right]}\end{array}$ & $R_{21}$ \\
\hline N1 & $(0,0)$ & 1.14 & 2.11 & 1.85 \\
N5 & $(0,0)$ & 1.52 & 0.70 & 0.46 \\
& $(10,0)$ & 1.27 & 1.05 & 0.83 \\
& $(-10,0)$ & 1.39 & 0.70 & 0.50 \\
& $(20,0)$ & 0.89 & 0.53 & 0.59 \\
& $(-20,0)$ & 1.52 & 1.05 & 0.69 \\
& $(20,-10)$ & 2.03 & 1.05 & 0.52 \\
N7 & $(0,0)$ & 2.53 & 1.23 & 0.49 \\
& $(20,0)$ & 1.52 & 1.93 & 1.27 \\
& $(-20,0)$ & 2.28 & 1.05 & 0.46 \\
& $(0,20)$ & 0.51 & 1.40 & 2.77 \\
& $(0,-20)$ & 1.39 & 4.04 & 2.90 \\
S2 & $(-10,0)$ & 0.76 & 1.05 & 1.39 \\
& $(0,-10)$ & 2.03 & 2.28 & 1.13 \\
& $(20,0)$ & 2.03 & 1.40 & 0.69 \\
& $(-20,0)$ & 1.65 & 1.40 & 0.85 \\
\hline
\end{tabular}

emission of a sample of 44 nearby spiral galaxies - to study the molecular gas emission in the centers and inner disks of galaxies in their sample with a resolution of $6^{\prime \prime}$. Their CO maps display a remarkable variety of molecular gas morphologies, and the CO surface brightness distribution shows substructures with dimensions of a few hundred parsecs. Sakamoto et al. (1999) found a similar result with a $\mathrm{CO}$ aperture-synthesis of the central regions of 20 nearby spiral galaxies using the Nobeyama and Owens Valley millimetric arrays with a typical resolution of $4^{\prime \prime}$. In many galaxies they detected CO clumps of subkiloparsec size that they defined as giant molecular associations (GMAs).

In Figs. 5 and 6 it appears difficult to distinguish individual structures, such as molecular clouds - probably present - and estimate their characteristic parameters (dimension, mass, etc.). To study the molecular gas structure on a small scale, it is necessary to use specific tools to identify single CO clouds.
There are several different methods of decomposing clumps in a 3-dimensional space; i.e. position-position-velocity space, and all the techniques developed allow characterization of the clumpy structure: size, line width, and one of the most important parameters of a clump, the mass. The algorithms generally used to study the clumpy nature of the gas are GAUSSCLUMPS (Stutzki \& Guesten 1990; Kramer et al. 1998) and GAUSSFIND (Williams et al. 1994). GAUSSCLUMPS decomposes the 3-dimensional data cubes into a series of clumps, where each clump corresponds to a peak position and is assumed to have a Gaussian shape. GAUSSFIND works on the 3-dimensional data cubes as the eye would find a data set by using local maxima. Usually, the results of the two algorithms agree with each other. In general, GAUSSCLUMPS is able to more easily find low-mass clumps than GAUSSFIND; and since M 81 shows a weak CO emission, we decided to use GAUSSCLUMPS. In addition, this algorithm can work with the package GILDAS, already used here.

\subsection{Identification of molecular associations}

The first application of GAUSSCLUMPS to $\mathrm{CO}(2-1)$ data obtained using HERA receiver found 27 molecular associations in the central $1.3 \mathrm{kpc}$; however, it is necessary to fix the constraints to check misidentification. The algorithm forces the unresolved clumps to have a size corresponding to the spatial and velocity resolutions, so if the $3 \mathrm{D}$ size of a clump is exactly equal to the resolution in each axis, this clump could be an artefact. With a beam of $12^{\prime \prime}$, only clumps with the intrinsic FHWM size $\Delta \xi>10^{\prime \prime}$ are realistic, so all the other clumps have been discarded. The second constraint is on the intrinsic brightness temperature, which must be at least 5 times the noise level of the original map, $T>0.035 \mathrm{~K}$ (Stutzki \& Guesten 1990). After applying these constraints, the number of molecular associations found in the M 81 central region has become 16 and their properties are listed in Table 4. In this table, Col. (1) is the GMA number; Cols. (2) and (3) are the GMA center positions referred to 
Table 4. GMAs identified in the M 81 center with GAUSSCLUMPS.

\begin{tabular}{|c|c|c|c|c|c|c|c|c|c|c|}
\hline Number & $\begin{array}{l}\Delta \alpha \\
{\left[{ }^{\prime \prime}\right]} \\
(2)\end{array}$ & $\begin{array}{l}\Delta \delta \\
{\left[{ }^{\prime \prime}\right]} \\
(3)\end{array}$ & $\begin{array}{c}v_{0} \\
{\left[\mathrm{~km} \mathrm{~s}^{-1}\right]} \\
(4)\end{array}$ & $\begin{array}{c}T_{0} \\
{[\mathrm{~K}]} \\
(5)\end{array}$ & $\begin{array}{l}\Delta x_{1} \\
{\left[{ }^{\prime \prime}\right]} \\
(6)\end{array}$ & $\begin{array}{l}\Delta x_{2} \\
{\left[{ }^{\prime \prime}\right]} \\
(7)\end{array}$ & $\begin{array}{c}\varphi \\
{\left[^{\circ}\right]} \\
(8)\end{array}$ & $\begin{array}{c}\Delta v \\
{\left[\mathrm{~km} \mathrm{~s}^{-1}\right]} \\
(9)\end{array}$ & $\begin{array}{c}\mathrm{d} v / \mathrm{d} r \\
{\left[\mathrm{~km} \mathrm{~s}^{-1}\right]} \\
(10)\end{array}$ & $\begin{array}{c}\varphi_{v} \\
{\left[{ }^{\circ}\right]} \\
(11)\end{array}$ \\
\hline 1 & 6.9 & 20.0 & 6.13 & 0.07 & 15.8 & 19.2 & 83.7 & 17.2 & 1.67 & 88.2 \\
\hline 2 & 9.4 & 8.8 & 238.31 & 0.06 & 12.0 & 12.1 & 5.9 & 12.1 & 2.76 & -111.8 \\
\hline 3 & -14.8 & -16.1 & 133.76 & 0.05 & 12.0 & 12.1 & 101.3 & 12.1 & 2.29 & -106.3 \\
\hline 4 & -7.1 & 27.3 & 132.12 & 0.05 & 12.0 & 17.2 & 94.5 & 13.9 & 2.05 & -69.7 \\
\hline 5 & 4.4 & -20.2 & 341.63 & 0.05 & 12.0 & 12.1 & 36.9 & 12.1 & 1.72 & -138.2 \\
\hline 6 & 8.9 & 2.7 & 524.09 & 0.05 & 12.0 & 19.0 & 43.8 & 14.3 & 2.04 & -90.2 \\
\hline 7 & -17.3 & -20.6 & -21.47 & 0.04 & 12.0 & 17.2 & 48.5 & 13.9 & 1.40 & -86.3 \\
\hline 8 & 9.2 & -28.2 & 264.09 & 0.04 & 12.0 & 12.1 & 56.9 & 12.1 & 3.04 & -149.6 \\
\hline 9 & -15.7 & -20.2 & 185.01 & 0.04 & 12.0 & 16.8 & 65.1 & 13.8 & 1.69 & 44.6 \\
\hline 10 & -14.7 & -27.0 & 263.61 & 0.04 & 12.0 & 12.1 & 63.6 & 12.1 & 4.41 & 28.6 \\
\hline 11 & 7.9 & 9.0 & 30.24 & 0.04 & 12.0 & 13.0 & 121.2 & 12.5 & 3.42 & -33.7 \\
\hline 12 & 1.6 & 27.2 & 56.42 & 0.04 & 12.0 & 23.6 & 88.8 & 15.1 & 1.39 & -105.3 \\
\hline 13 & 8.4 & -15.5 & 316.24 & 0.04 & 12.0 & 12.1 & 87.7 & 12.1 & 5.83 & 4.7 \\
\hline 14 & 9.2 & -14.9 & 82.50 & 0.04 & 12.0 & 12.1 & 22.6 & 12.1 & 3.62 & 115.8 \\
\hline 15 & -32.9 & -9.2 & -22.05 & 0.04 & 12.9 & 20.0 & 81.0 & 15.3 & 1.04 & -18.8 \\
\hline 16 & -14.5 & 21.9 & -22.41 & 0.04 & 19.0 & 12.1 & 29.3 & 14.5 & 2.18 & 42.0 \\
\hline
\end{tabular}

the center of the galaxy, which coordinates are given in Table 1; Col. (4) is the GMA center velocity; Col. (5) is the GMA peak intensity; Cols. (6) and (7) are the GMA spatial FWHMs along the two principal axis; Col. (8) is the orientation of the first principal axes; Col. (9) is the GMA velocity FWHM; Col. (10) is the GMA internal velocity gradient; Col. (11) is the direction of internal velocity gradient.

Considering that the Gaussian-shaped molecular associations have two principal axes, we find a mean intrinsic diameter for all molecular structures identified of $14^{\prime \prime}(\sim 250 \mathrm{pc})$. Molecular structures with these dimensions are defined as GMAs. The maximum GMA peak intensity is $0.070 \mathrm{~K}$, the mean intensity is $0.046 \mathrm{~K}$ and the mean velocity FWHM $13.5 \mathrm{~km} \mathrm{~s}^{-1}$.

Summing up the area corresponding to the intrinsic FWHM extent of the fitted GMAs in the selected field, one obtains a total area covered by GMAs that is from 2.2 to 4.5 times smaller than the field, therefore the filling factor of the GMAs is low, about 0.3 .

\subsection{GMAs masses}

For an optically thin gas, or at the other extreme for a completely optically thick gas (Kramer et al. 1998), the mass of a molecular association is proportional to the fitting parameters of GAUSSCLUMPS: the molecular association intensity, the molecular association velocity width, and the spatial FWHMs along both two principal axes:

$M \propto T_{\mathrm{pk}} \times \Delta v \times \Delta x_{1} \times \Delta x_{2}$

We now present mass computations in the two hypotheses below. For the estimation in the optically thin case, we adopt the Rayleigh-Jeans approximation, where: $f\left(T_{\mathrm{ex}}\right)=\frac{h v / k}{1-\exp \left[-h v / k T_{\mathrm{ex}}\right]} \sim$ $T_{\mathrm{ex}}$, since $T_{\mathrm{ex}}>15 \mathrm{~K}$. LTE is also assumed, meaning that $T_{\text {ex }}$ is the same for all levels. The total CO column density $N_{\mathrm{CO}}$ is related to the population $N_{i}$ of level $i$ by $N_{i} / N_{\mathrm{CO}}=$ $g_{i} \exp \left[-E_{i} / k T_{\text {ex }}\right] / Q$, where $g_{i}$ and $E_{i}$ are the degeneracy factor and the energy of the level $i$, and $Q$ the partition function is $Q(T)=2 k T / h v_{1-0}=T(K) / 2.76$. This total CO column density, derived from the integrated intensity of the $\mathrm{CO}(2-1)$ line, is given by the following equation, in the optically thin limit:

$\frac{N_{\mathrm{CO}}}{\left(\mathrm{mol} / \mathrm{cm}^{2}\right)}=1.2 \times 10^{13} \times T_{\mathrm{ex}}(\mathrm{K}) \int T_{\mathrm{mb}}(K) \mathrm{d} v\left(\mathrm{~km} \mathrm{~s}^{-1}\right)$

The corresponding molecular mass (taking into account helium) is

$$
\begin{aligned}
\frac{M}{\left(M_{\odot}\right)}= & 1.2 \times 10^{13} \frac{m_{\mathrm{H}_{2}}}{\left(M_{\odot}\right)} \frac{N\left(\mathrm{H}_{2}\right)}{N\left({ }^{12} \mathrm{CO}\right)} \times T_{\mathrm{ex}}(\mathrm{K}) \\
& \times \iint T_{\mathrm{mb}}(\mathrm{K}) \mathrm{d} v\left(\mathrm{~km} \mathrm{~s}^{-1}\right) \mathrm{d} A\left(\mathrm{~cm}^{2}\right) .
\end{aligned}
$$

In terms of fitting parameters in Table 4 (see Chi \& Park 2006) and when assuming an excitation temperature of $T_{\mathrm{ex}} \simeq 15 \mathrm{~K}$, an abundance ratio $N\left(\mathrm{H}_{2}\right) / \mathrm{N}\left({ }^{12} \mathrm{CO}\right) \simeq 10^{4}$, and a distance of 3.6 Mpc, Eq. (3), valid in the optically thin case, becomes

$\frac{M_{\mathrm{thin}}}{\left(M_{\odot}\right)}=\frac{T_{\mathrm{mb}}}{(K)} \times \frac{\Delta v}{\left(\mathrm{~km} \mathrm{~s}^{-1}\right)} \times \frac{\Delta x_{1}}{\left({ }^{\prime \prime}\right)} \times \frac{\Delta x_{2}}{\left({ }^{\prime \prime}\right)} \times 10.5$.

In this hypothesis, the ratio between the observed peak brightness temperature and the excitation temperature is the filling factor of the porous molecular associations, on the order of $3 \times 10^{-3}$. Applying Eq. (4), we find very small molecular association masses contained in a low mass range, from $7.37 \times 10^{2} M_{\odot}$ to $3.83 \times 10^{3} M_{\odot}$, with a mean value of $1.31 \times 10^{3} M_{\odot}$. The masses computed for all molecular associations are listed in Table 5. In this table, Col. (1) is the GMA number as defined in Table 4; Col. (2) is the effective radius of the GMAs; Cols. (3), (4), and (5) are the masses of the GMAs computed in the optically thin limit, in the optically thick limit, and using the virial theorem, respectively; Col. (6) is the ratio $\alpha_{\mathrm{vir}}=M_{\mathrm{vir}} / M_{\mathrm{obs}}$ for each GMA, where $M_{\text {obs }}=M_{\text {thick }}$; Col. (7) is the derived $X$ conversion factor for each GMA.

In the optically thick case, the molecular mass of the molecular associations is determined by the $\mathrm{H}_{2}$ density $\left(N_{\mathrm{H}_{2}}\right)$ using the standard $X$ conversion factor value $\left(X=2.3 \times 10^{20} \mathrm{~mol} \mathrm{~cm}^{-2}\right.$ $\left(\mathrm{K} \mathrm{km} \mathrm{s}^{-1}\right)^{-1}$, Strong et al. 1988), $N_{\mathrm{H}_{2}}=X \int T_{\mathrm{CO}(1-0)} \mathrm{d} v$. Since we resolve single GMAs in the $\mathrm{CO}(2-1)$ transition, using the 
Table 5. Properties of the GMAs derived by application of GAUSSCLUMPS.

\begin{tabular}{|c|c|c|c|c|c|c|}
\hline Number & $\begin{array}{c}{[\mathrm{pc}]} \\
\text { (2) }\end{array}$ & $\begin{array}{c}M_{\text {thin }} \\
10^{3} \\
{\left[M_{\odot}\right]} \\
(3)\end{array}$ & $\begin{array}{c}M_{\text {thick }} \\
10^{5} \\
{\left[M_{\odot}\right]} \\
(4)\end{array}$ & $\begin{array}{c}M_{\mathrm{vir}} \\
10^{6} \\
{\left[M_{\odot}\right]} \\
(5)\end{array}$ & $\alpha_{\mathrm{vir}}$ & $\begin{array}{c}X \\
10^{21} \\
{\left[\mathrm{~mol} \mathrm{~cm}^{-2}\left(\mathrm{~K} \mathrm{~km} \mathrm{~s}^{-1}\right)^{-1}\right]} \\
(7)\end{array}$ \\
\hline 1 & 151.88 & 3.83 & 7.20 & 8.54 & 11.86 & 2.29 \\
\hline 2 & 105.08 & 1.11 & 2.08 & 2.92 & 14.07 & 2.71 \\
\hline 3 & 105.08 & 0.92 & 1.73 & 2.92 & 16.89 & 3.26 \\
\hline 4 & 125.28 & 1.50 & 2.83 & 4.60 & 16.27 & 3.14 \\
\hline 5 & 105.08 & 0.92 & 1.73 & 2.92 & 16.89 & 3.26 \\
\hline 6 & 131.67 & 1.71 & 3.21 & 5.12 & 15.93 & 3.07 \\
\hline 7 & 125.28 & 1.20 & 2.26 & 4.60 & 20.34 & 3.92 \\
\hline 8 & 105.08 & 0.74 & 1.38 & 2.92 & 21.11 & 4.07 \\
\hline 9 & 123.81 & 1.17 & 2.19 & 4.48 & 20.43 & 3.94 \\
\hline 10 & 105.08 & 0.74 & 1.38 & 2.92 & 21.11 & 4.07 \\
\hline 11 & 108.91 & 0.82 & 1.54 & 3.23 & 21.04 & 4.06 \\
\hline 12 & 146.74 & 1.79 & 3.37 & 6.36 & 18.86 & 3.64 \\
\hline 13 & 105.08 & 0.74 & 1.38 & 2.92 & 21.11 & 4.07 \\
\hline 14 & 105.08 & 0.74 & 1.38 & 2.92 & 21.11 & 4.07 \\
\hline 15 & 140.06 & 1.66 & 3.11 & 6.23 & 20.03 & 3.86 \\
\hline 16 & 132.22 & 1.40 & 2.63 & 5.28 & 20.10 & 3.88 \\
\hline
\end{tabular}

mean $R_{21}=I_{21} / I_{10}$ value computed here $\left(\bar{R}_{21}=0.68\right)$, the $\mathrm{H}_{2}$ density can be expressed by

$$
\begin{aligned}
\frac{N_{\mathrm{H}_{2}}}{\left(\mathrm{~mol} / \mathrm{cm}^{2}\right)} & =\frac{X}{\left(\frac{\mathrm{mol} / \mathrm{cm}^{2}}{\mathrm{~K} \mathrm{~km} \mathrm{~s}^{-1}}\right)} \times \frac{1}{R_{21}} \int T_{\mathrm{CO}(1-0)}(\mathrm{K}) \mathrm{d} v\left(\mathrm{~km} \mathrm{~s}^{-1}\right) \\
& =3.4 \times 10^{20} \int T_{\mathrm{CO}(1-0)}(\mathrm{K}) \mathrm{d} v\left(\mathrm{~km} \mathrm{~s}^{-1}\right),
\end{aligned}
$$

and the corresponding molecular mass is

$$
\begin{aligned}
\frac{M}{\left(M_{\odot}\right)}= & 3.4 \times 10^{20} \frac{m_{\mathrm{H}_{2}}}{\left(M_{\odot}\right)} \\
& \times \iint T_{\mathrm{CO}(1-0)}(\mathrm{K}) \mathrm{d} v\left(\mathrm{~km} \mathrm{~s}^{-1}\right) \mathrm{d} A\left(\mathrm{~cm}^{2}\right) .
\end{aligned}
$$

In terms of fitting parameters in Table 4 and assuming a distance of $3.6 \mathrm{Mpc}$, Eq. (6) gives the GMA mass in the optically thick limit:

$\frac{M_{\text {thick }}}{\left(M_{\odot}\right)}=\frac{T_{\mathrm{mb}}}{(\mathrm{K})} \times \frac{\Delta v}{\left(\mathrm{~km} \mathrm{~s}^{-1}\right)} \times \frac{\Delta x_{1}}{\left({ }^{\prime \prime}\right)} \times \frac{\Delta x_{2}}{\left({ }^{\prime \prime}\right)} \times 1.97 \times 10^{3}$.

Applying Eq. (7), the GMA masses range from $1.38 \times 10^{5} M_{\odot}$ to $7.20 \times 10^{5} M_{\odot}$, with a mean value of $2.46 \times 10^{5} M_{\odot}$. The total mass of the GMAs is $3.22 \times 10^{5} M_{\odot}$. The masses computed for all GMAs are listed in Table 5.

That the masses obtained through the optical thin hypothesis are much lower than those calculated in the optically thick case is not "anomalous": instead, it is expected that the optical thin hypothesis underestimates the masses, since GMAs have a rich substructure of subclumps with high column density. The subclumps are probably optically thick, as is generally the case in external galaxies, as suggested by the high ${ }^{13} \mathrm{CO}$ emission commonly observed. Anyway we decided to also analyze the optically thin case because M 81 represents an extraordinary laboratory where all possibilities should be considered even if unusual for similar galaxies.

\subsection{Virial equilibrium}

An alternative way of determining GMA masses is to compute their virial mass. Assuming a balance between the total kinetic energy $T$ of a GMA and the gravitational potential $\Omega$, and neglecting external pressure, the virial mass $M_{\text {vir }}$ of the clouds can be calculated using the formula

$M_{\text {vir }}=K \times D_{\mathrm{e}} \times \Delta v^{2}$

where $M_{\text {vir }}$ is in $M_{\odot}, D_{\mathrm{e}}$ is the effective diameter in pc of the equivalent sphere of radius $R=\left(\Delta x_{1} \Delta x_{2} / 4\right)^{0.5}$, and $\Delta v$ is in $\mathrm{km} \mathrm{s}^{-1}$ (Solomon et al. 1987). We assume that the cloud is a sphere with a power-law $(\alpha=1)$ density distribution. In this case, the constant $K$ in formula (8) is equal to 95 . The rougher assumption of uniform density (8) would have instead produced $K=104$. Here, the hypothesis of spherical shape for the cloud makes the formula independent of the galaxy inclination.

A comparison between the virial mass and the mass computed from the observed intensity lines $\left(M_{\mathrm{obs}}\right)$ in the optical thick hypothesis is informative for the physical state of the clouds, their optical thickness, and for the actual $X=N\left(\mathrm{H}_{2}\right) / I(\mathrm{CO})$ conversion factor.

The virial masses of the 16 GMAs we identified span from $2.92 \times 10^{6}$ to $8.54 \times 10^{6} M_{\odot}$, with a mean value of $4.31 \times 10^{6} M_{\odot}$. In calculating $M_{\text {vir }}$, we have neglected the mass of the atomic hydrogen $\left(M_{\mathrm{HI}}=0\right)$ since Allen et al. (1997), studying the HI profile in $M$ 81, found a deficiency of atomic hydrogen in the central $\sim 3 \mathrm{kpc}$, and the HI emission only begins to be significant in the inner spiral arms beyond $\sim 5 \mathrm{kpc}$ from the nucleus.

We also checked that the stars do not contribute significantly to the mass inside the volume of a GMA. In the hypothesis that the $\mathrm{H}_{2}$ mass is computed from the virial mass, resolved GMAs have mean surface densities ranging from $84 M_{\odot} / \mathrm{pc}^{2}$ to $118 M_{\odot} / \mathrm{pc}^{2}$ with a mean value of $91 M_{\odot} / \mathrm{pc}^{2}$. The mean value of the mass-to-light ratio of visible matter expected for $M 81$ is $M / L_{B}=5.5 M_{\odot} / L_{\odot}$ (see Tenjes 1994). From the exponential surface brightness distribution of the stellar disk of the galaxy, we then deduced a mean stellar surface density of $36 M_{\odot} / \mathrm{pc}^{2}$ in the region. In addition, if we consider that GMAs normally have a thickness of about $50 \mathrm{pc}$ while the stars are distributed in regions that are at least ten times thicker, the actual mean volumic stellar density is negligible with respect to the molecular density inside a GMA.

All the GMAs have a virial mass $\gg$ of the mass computed from CO observations, and the mean ratio $\alpha_{\mathrm{vir}}=M_{\mathrm{vir}} / M_{\mathrm{obs}}$ 
computed for our GMAs is $\alpha_{\text {vir }} \simeq(10-20) \gg 1$. The $\alpha_{\text {vir }}$ ratios computed for all the GMAs are listed in Table 5.

\subsection{The $\mathrm{N}\left(\mathrm{H}_{2}\right) / \mathrm{I}_{\text {CO }}$ conversion factor}

The value of the conversion factor between the $\mathrm{H}_{2}$ column density of the molecular hydrogen $N\left(\mathrm{H}_{2}\right)$ and the $\mathrm{CO}$ integrated intensity $\left(X=N\left(\mathrm{H}_{2}\right) / I_{\mathrm{CO}}\right)$ is still subject to debate. Usually the standard value of $\mathrm{X}$ found for the Milky Way is universally adopted for all the galaxies and for all the regions of the same galaxy $\left(X=2.3 \times 10^{20} \mathrm{~mol} \mathrm{~cm}{ }^{-2}\left(\mathrm{~K} \mathrm{~km} \mathrm{~s}^{-1}\right)^{-1}\right.$, Strong et al. 1988). This assumption often turned out not to be completely correct.

The $X$ conversion factor is determined by various factors, such as the metallicity, the temperature, the cosmic ray density, and the UV radiation field (see Maloney \& Black 1988; Boselli et al. 2002). For instance, galaxies with lower metallicity generally have a higher $X$ factor; when studying 5 local group galaxies, Wilson (1995) found that the conversion factor increases by a factor of 4.6 when metallicity decreases of a factor 10 . The $X$ value also changes with the morphological type of the galaxies: galaxies earlier than Scd show values comparable to, or lower than, the Galactic one, while extremely late-type spirals or irregular galaxies tend to show higher values (Nakai \& Kuno 1995). A dependency of the $X$ conversion factor on $I_{\mathrm{CO}}$ is also suspected: galaxies or observed positions with low $I_{\mathrm{CO}}\left(I_{\mathrm{CO}}<20-\right.$ $40 \mathrm{~K} \mathrm{~km} \mathrm{~s}^{-1}$ ) show a higher $X$. An example of this behavior is the galaxy M 51, where $I_{\mathrm{CO}}<20 \mathrm{~K} \mathrm{~km} \mathrm{~s}^{-1}$ and the $X$ derived value is a factor 2.6 lower than the mean Galactic value (see Fig. 3 of Nakai \& Kuno 1995).

Since M 81 shows atypical properties for its molecular gas, the $\mathrm{CO}$ to $\mathrm{H}_{2}$ conversion ratio is also suspected of departing significantly from the mean Galactic value and in general from that of galaxies with the same morphological type. Thus, M 81 represents an exceptional laboratory to explore the variation of the $X$-ratio.

We derived the $X$ conversion factor for all individually resolved molecular associations assuming the virial equilibrium for single molecular complexes and using the large velocity gradient (LVG) approximation for the formation of the CO line (Young \& Scoville 1984; Sanders et al. 1984). This method has also been used to derive the conversion factor in nearby spiral galaxies such as M 31, M 33, and the Magellanic Clouds (Wilson \& Scoville 1990, 1992; Arimoto et al. 1996).

In practice, we computed the $\mathrm{X}$ ratio $\left(=N\left(\mathrm{H}_{2}\right) / I_{\mathrm{CO}}\right)$ deriving the $\mathrm{H}_{2}$ density from the virial masses $\left(N\left(\mathrm{H}_{2}\right)=M_{\mathrm{vir}} /\left(m_{\mathrm{H}_{2}} \times \mathrm{d} A\right)\right.$, where $m_{\mathrm{H}_{2}}$ is the mass of the hydrogen molecule and $d A$ the area occupied by the cloud), and $I_{\mathrm{CO}}$ is the $\mathrm{CO}$ observed intensity. For the individually resolved GMAs of M 81, assumed virialized, we found that the $X$ conversion factor takes a much higher value than the mean Galactic value for all clouds, ranging from $\sim 10$ to $\sim 18$ times higher than the standard $X$ and a mean value of $X=3.6 \times 10^{21} \mathrm{~mol} \mathrm{~cm}^{-2}\left(\mathrm{~K} \mathrm{~km} \mathrm{~s}^{-1}\right)^{-1}$. The $X$ conversion factor values computed for all the GMAs are listed in Table 5.

Our $X$ factor values are high, not only if compared to the standard Galactic factor, but also with respect to values found in other nearby galaxies where the $\mathrm{X}$ ratio has been obtained with the same method we used. Studying a sample of nearby spiral and dwarf irregular galaxies, Arimoto et al. (1996) derived $X$ values using $\mathrm{CO}$ data found in the literature and computed the corresponding virial mass. They found galaxies with both $X$ values lower and higher than in the Galaxy, such as M 31 with $X=1.8 \times 10^{20} \mathrm{~mol} \mathrm{~cm} \mathrm{~cm}^{-2}\left(\mathrm{~K} \mathrm{~km} \mathrm{~s}^{-1}\right)^{-1}$, M 33 with $X=4.1 \times 10^{20} \mathrm{~mol} \mathrm{~cm}^{-2}\left(\mathrm{~K} \mathrm{~km} \mathrm{~s}^{-1}\right)^{-1}$, and IC 10 where
$X=6.6 \times 10^{20} \mathrm{~mol} \mathrm{~cm}^{-2}\left(\mathrm{~K} \mathrm{~km} \mathrm{~s}^{-1}\right)^{-1}$. Even if they found high $\mathrm{X}$ values, these $\mathrm{H}_{2}$-to-CO conversion factors are smaller than our X ratios obtained for M 81. Arimoto et al. (1996) found a $X$ ratio $\left(=3.1 \times 10^{21} \mathrm{~mol} \mathrm{~cm}^{-2}\left(\mathrm{~K} \mathrm{~km} \mathrm{~s}^{-1}\right)^{-1}\right)$ only for the SMC within the same order of magnitude of $M$ 81. They specify that this value has been obtained by considering virial masses and CO luminosities for larger-scale complexes in the SMC and that this high $X$ would be due to a smaller amount of $\mathrm{CO}$ in a diffuse medium that is metal dependent, as well as photo-dissociated by the strong UV field, and can be present only in dense molecular clouds.

\section{Discussion}

\subsection{Comparison with previous works}

Our molecular gas detections in the center of M 81 agree with the only two works present in the literature that report CO emission in the nuclear region of this galaxy (Sage \& Westpfahl 1991; Sakamoto et al. 2001). In comparison with the giant molecular clouds (GMCs) in the outer regions of M 81 (Taylor \& Wilson 1998; Brouillet et al. 1998), the CO emission that we detect in the M 81 center is particularly cold and unusual. The masses we find for GMAs in the $\mathrm{CO}(2-1)$ map are similar to the higher masses observed in M 33 (Wilson \& Scoville 1990), and higher than what is measured in the SMC (Rubio et al. 1993).

Our first result is that the nuclear region of M 81 (within $\sim 300$ pc) appears devoid of CO emission, in agreement with Brouillet et al. (1988) and Sakamoto et al. (2001). However, we detect $\mathrm{CO}$ emission outside the $300 \mathrm{pc}$ radius. This supports the claims of Sage \& Westpfahl (1991) who found CO emission when observing one offset towards the center galaxy with the NRAO $12 \mathrm{~m}$ telescope and a beam of $55^{\prime \prime}$ (see Fig. 1 of Sage \& Westpfahl 1991). There is a good correspondence between our velocity profile and theirs.

A more useful and interesting comparison can be done between our results and those of Sakamoto et al. (2001). In detail, the "pseudoring" that we detected at $\sim 460 \mathrm{pc}$ from the nucleus in the northeast direction (Fig. 3) corresponds to the maximum intensity of Sakamoto et al. (2001). Observing the central kiloparsec with the Nobeyama Millimetric Array-NMA (resolution of $6.9^{\prime \prime} \times 5.8^{\prime \prime}$ that corresponds to $\sim 120 \times 100 \mathrm{pc}$ at the distance of M 81), they produced maps only for the ${ }^{12} \mathrm{CO}(1-0)$ line. Since they made interferometric measurements, with a FWHM primary beam of $1^{\prime}$ and a beam of $\sim 7^{\prime \prime}$, extended emission seen with the $22^{\prime \prime}$ IRAM CO $(1-0)$ beam is filtered out.

In spite of this, the total mass of molecular gas that we find, assuming the same standard X-factor value of Strong et al. (1988), is quite comparable to that of Sakamoto et al. (2001): our resolved GMAs in $\mathrm{CO}(2-1)$ emission have a total mass of $3.22 \times 10^{6} M_{\odot}$, only a little bit lower than $\sim 1 \times 10^{7} M_{\odot}$ found by Sakamoto et al. (2001) for the "pseudoring" in CO(1-0) emission. In addition to the result of Sakamoto et al. (2001), who found the molecular gas mainly located in a lopsided distribution in the northeast direction, we also detected some emission towards the southwest. Our peak temperatures for the ${ }^{12} \mathrm{CO}(1-$ 0 ) line are very low, ranging from $\sim 15$ to $\sim 64 \mathrm{mK}$, lower than the average peak brightness temperature $(\sim 0.3 \mathrm{~K})$ of each GMA found by Sakamoto et al. (2001). This is compatible with the different beam sizes, the synthesized beam of the interferometer being 10 times smaller in surface.

Another useful comparison can be made with molecular clouds identifications in other external galaxies. The masses $\left(\sim 10^{5} M_{\odot}\right)$ and the diameters $(\sim 250 \mathrm{pc})$ of the CO clouds 
identified in M 81 are not much unusual for giant galaxies of the same type (Taylor \& Wilson 1998). Even if in general the GMCs and GMAs in the galaxies of the Local Group are very similar, there is evidence of variations in the properties of the molecular clouds of galaxies of different morphologies, which range from early-type spirals (M 31 and M 81) to late-type spirals (M 33 of Scd morphological type) and from dwarf irregulars (IC 10) to dwarf ellipticals (NGC 205). For the variety of characteristics found in clouds of galaxies of these sub-morphological types, Taylor \& Wilson (1998) conclude that in general very late-type galaxies should have smaller and less massive GMCs than earlytype galaxies. Our results agree with this conclusion.

Finally, we know that, in the Local Group and in systems in interaction with the Local Group, macroscopic properties of molecular clouds are related by power relationships, first noted by Larson (1981) and then studied in detail by Solomon et al. (1987). In particular, Solomon et al. (1987) found a tight relationship between the Galactic dynamical molecular cloud masses, measured by the virial theorem, and the $\mathrm{CO}$ luminosity $\left(M_{\mathrm{vir}} \propto\left(L_{\mathrm{CO}}\right)^{0.81}\right)$. For several external galaxies, this power law is even valid if with little variations of the power index (Rand \& Kulkarni 1990; Rand 1995; Rosolowsky \& Blitz 2005). It is interesting to test if M 81 also shows non-typical behavior in this case. Our very weak CO detections correspond to CO luminosities of the order of magnitude $10^{4} \mathrm{~K} \mathrm{~km} \mathrm{~s}^{-1} \mathrm{pc}^{2}$ with virial masses within an order of magnitude of $10^{6} M_{\odot}$ (see Table 5). Comparing our results with the Fig. 2 of Solomon et al. (1987), we can see that with the $L_{\mathrm{CO}}=10^{4} \mathrm{~K} \mathrm{~km} \mathrm{~s}^{-1} \mathrm{pc}^{2}$ virial masses we infer are overestimated by more than one order of magnitude with respect to the typical trend in our Galaxy, but also in external galaxies. This result agrees with the indications given by the $\alpha_{\text {vir }}$ ratios calculated in Sect. 4.3, and in general it confirms the unusual scenario in the molecular gas properties of M 81 .

\subsection{The $X$-factor problem}

The $X$-factor peculiarity does not appear to be due to a particular metallicity, since $M 81$ has a metallicity of $Z=0.03$, without any evident gradient from the central region to the outer disk (Kong et al. 2000). This "normal" metallicity value would produce a $X$ conversion factor similar to that of the Milky Way. Some regions in M 81, however, have higher metallicity and are mostly located in the spiral arms and HII regions. But in general, our estimate of the $X$-factor appears significantly high.

A possible source of error may be the assumption of a fully virial equilibrium for the observed clouds. Under this hypothesis, we calculated the $X$-factor using $X=M_{\text {vir }} / L_{\mathrm{CO}}$, where the $M_{\text {vir }}$ has been computed with Eq. (8), and $L_{\mathrm{CO}}$ is the CO luminosity derived from our observations. Application of the virial theorem to complex molecular structures may not be completely appropriate, even if the expected deviation cannot be higher than a factor $\sim 2$. However, if the GMAs are not virialized and the standard $X$ factor is applied, the molecular content of M 81 would be exceptionally low, so M 81 would be peculiar in both cases.

\subsection{Heating of the gas}

The reason for the non-typical results on the molecular gas in M 81 is probably the exceptionally low $\mathrm{CO}$ excitation temperature that seems to characterize the center of this galaxy. Why is the molecular gas absent or subthermally excited in the M 81 center?
A possible link could exist between low $\mathrm{CO}$ emission and the lack of ultraviolet emission that appears to characterize the M 81 central region. The ultraviolet image of M 81 realized with the satellite Galaxy Evolution Explorer (GALEX) clearly shows a lack of recent star formation, traced by the UV emission, in the nuclear region that we observed in $\mathrm{CO}$.

The existence of a link between molecular gas and farultraviolet (FUV) emission can be justified considering that the interstellar medium is excited, dissociated, and ionized by the FUV photons produced by young $\mathrm{O}$ and B stars. Atomic gas in the ISM recombines into molecular form mainly through the catalytic action of dust grain surfaces. Hydrogen nuclei cycle repeatedly from the molecular $\left(\mathrm{H}_{2}\right)$ to the atomic phase and back again at rates that depend on the incident FUV flux, the total volume density of the gas, and the dust-to-gas ratio. The action of the FUV flux that interacts with the surface of the molecular clouds can strongly affect their physics and chemistry by heating, dissociating, and exciting the gas.

In regions of the ISM where the physics is dominated by FUV photons - called photodissociation regions (PDRs) - useful information can be obtained on the physical state of the gas from observations of the spectral lines emitted by the excited atoms and molecules in those regions.

In M 81 the possibility that low CO emission and weak FUV flux are correlated has already been proposed by Knapen et al. (2006). When observing a region of the western spiral arm in M 81, they explained the lack of or very weak $\mathrm{CO}(1-0)$ emission, observed far from FUV sources, as a consequence of insufficient heating and excitation of the molecular gas. Usually, a lack in the $\mathrm{CO}$ emission is instead interpreted as due to the absence of measurable quantities of molecular gas or due to a low density gas not heated sufficiently to detect it. The limit of this interpretation, based on the linear proportionality between the $\mathrm{CO}$ surface brightness and the column density of the molecular hydrogen, is to neglect effects linked to local heating mechanisms. If the explanation of Knapen et al. (2006) is correct, the flux of cosmic rays and the FUV surface brightness would be too low to heat the ISM, and in particular, the molecular component. It is possible that this correlation of low-FUV/low-CO is also applicable to the central region that we observed.

To verify this interpretation, we performed aperture photometry in the FUV emission on three fields already observed in $\mathrm{CO}$ : our central region of $1.6 \mathrm{kpc}$, the field of spiral arms observed by Brouillet et al. (1998), and that - again on the spiral arms - observed by Knapen et al. (2006). The fields selected on the spiral arms are the same of Fig. 2 of Knapen et al. (2006). We used the FUV-GALEX image of M 81 expressed in intensity and already sky-subtracted and performed the aperture photometry using the task QPHOT of IRAF, able to do a quick aperture photometry. This procedure allowed us to know the FUV flux emitted in regions observed in $\mathrm{CO}(1-0)$ and hence to compute the ratio between the two emissions in different fields, but of similar dimensions, of the galaxy. The aperture photometry was done with a radius of $40^{\prime \prime}$ for our central region and for the one observed by Knapen et al. (2006) with the Nobeyama Radio Observatory (NRO), and with a radius of $32^{\prime \prime}$ for the field observed by Brouillet et al. (1998). The results of the aperture photometry are summarized in Table 6. In this table, Col. (1) gives the references relatively to $\mathrm{CO}(1-0)$ emission; Col. (2) is the $\mathrm{CO}(1-0)$ integrated intensity; Col. (3) is the FUV flux obtained by the aperture photometry; Col. (4) is FUV flux expressed in terms of the $G_{0}$ Habing unit; Col. (5) is the ratio between the $\mathrm{CO}(1-0)$ integrated intensity and the FUV flux. We express the FUV fluxes in terms of the Habing unit, since it is 
Table 6. Comparison of the results obtained applying the aperture photometry at the FUV-GALEX image of M 81 in different regions observed in $\mathrm{CO}(1-0)$.

\begin{tabular}{|c|c|c|c|c|}
\hline Reference & $\begin{array}{c}I_{\mathrm{CO}} \\
{\left[\mathrm{K} \mathrm{km} \mathrm{s}^{-1}\right]} \\
\end{array}$ & $\begin{array}{c}F_{\mathrm{FUV}} \\
10^{-14} \\
{\left[\mathrm{erg} \mathrm{cm}^{-2} \mathrm{~s}^{-1} \AA^{-1}\right]}\end{array}$ & $G_{0}$ & $\begin{array}{c}I_{\mathrm{CO}} / F_{\mathrm{FUV}} \\
10^{14} \\
{\left[\mathrm{~K} \mathrm{~km} \mathrm{~s}^{-1} /\left(\mathrm{erg} \mathrm{cm}^{-2} \mathrm{~s}^{-1} \AA^{-1}\right)\right]} \\
\end{array}$ \\
\hline Brouillet et al. (1998) & 25.90 & 1.37 & 0.51 & 18.88 \\
\hline Knapen et al. (2006) & 20.26 & 1.48 & 0.35 & 13.65 \\
\hline This paper & 53.50 & 1.13 & 0.31 & 47.18 \\
\hline
\end{tabular}

one of the units usually used to model PDRs (e.g. Kaufman et al. 1999; Allen et al. 2004). In these models each one-dimensional semi-infinite slab of gas with constant density of Huclei is subjected to an equivalent one-dimensional flux of FUV photons $G_{0}$ measured in units of $1.6 \times 10^{-3} \mathrm{erg} \mathrm{cm}^{-2} \mathrm{~s}^{-1}$ over the photon energy range 6-13.6 eV. In these units, the average interstellar radiation field over $4 \pi$ sr is $G_{0}=1.7$ (Draine 1978; Allen et al. 2004).

The central region emits a weak FUV flux, within the same order of magnitude as that emitted in the two fields of the spiral arms, and the higher ratio between CO and FUV emissions in nuclear region is mainly due to the higher molecular gas intensity. If the low FUV emission is not able to heat the molecular gas sufficiently in the spiral arms, in the central region at the same quantity of FUV flux corresponds a molecular gas distributed in substructures, but subexcited. Probably the central region shows a higher $\mathrm{CO} / \mathrm{FUV}$ ratio because in the spiral arms the molecular gas is less excited than the nucleus.

\section{Conclusions}

The physics of the molecular gas suggested by the observations reported in this paper shows M 81 to be a galaxy that is not only $\mathrm{CO}$-poor in its central region, but that also has molecular clouds with unusual properties, if compared to galaxies with similar distances and morphological type. The absent or very weak molecular gas emission in the nuclear region, the low $R_{21}$ line ratio, and a particularly high $X$ conversion factor value are its main peculiarities. The $N\left(\mathrm{H}_{2}\right) / I_{\mathrm{CO}}$ conversion factor has a mean value of $X=3.6 \times 10^{21} \mathrm{~mol} \mathrm{~cm}^{-2}\left(\mathrm{~K} \mathrm{~km} \mathrm{~s}^{-1}\right)^{-1}, 15.6$ times higher than the $X$ value of Strong et al. (1988) derived for our Galaxy.

All unusual results found for the molecular component in the M 81 center are probably due to an excitation process of the gas non-typical for giant spirals in the Local Universe. The spiral arms of this galaxy are CO-poor, and it has been suggested that the FUV surface brightness is too low to heat the molecular gas component. The low $\mathrm{CO}$ emission we found in the center suggests that the gas in the nucleus is also subexcited, with even weaker FUV emission. We conclude that the lack of excitation of the gas, more than the absence of molecular gas, is the cause of the low $\mathrm{CO}$ emission there.

Acknowledgements. The observations reported here were made using the travel funds of RadioNet Networking Activities. V. Casasola is pleased to acknowledge the hospitality and stimulating environment provided by the Observatoire de Paris-LERMA, where part of the work on this paper was done during her stay in Paris, thanks to Grant Vinci and the EARA agreement. The authors would like to thank the anonymous referee, whose comments have been useful for improving the original version of the paper.

\section{References}

Allen, R. J., \& Lequeux, J. 1993, ApJ, 410, L15

Allen, R. J., Knapen, J. H., Bohlin, R., \& Stecher, T. P. 1997, ApJ, 487, 171

Allen, R. J., Heaton, H. I., \& Kaufman, M. J. 2004, ApJ, 608, 314

Arimoto, N., Sofue, Y., \& Tsujimoto, T. 1996, PASJ, 48, 275
Beck, R., Krause, M., \& Klein, U. 1985, A\&A, 152, 237

Bietenholz, M. F., Bartel, N., Rupen, M. P., et al. 1996, ApJ, 457, 604

Boselli, A., Lequeux, J., \& Gavazzi, G. 2002, Ap\&SS, 281, 127

Braine, J., \& Combes, F. 1992, A\&A, 264, 433

Braine, J., \& Combes, F. 1993, A\&A, 269, 7

Brouillet, N., Baudry, A., \& Combes, F. 1988, A\&A, 196, L17

Brouillet, N., Baudry, A., Combes, F., Kaufman, M., \& Bash, F. 1991, A\&A, 242, 35

Brouillet, N., Kaufman, M., Combes, F., Baudry, A., \& Bash, F. 1998, A\&A, 333, 92

Casasola, V., Bettoni, D., \& Galletta, G. 2004, A\&A, 422, 941

Casoli, F., Clausset, F., Combes, F., Viallefond, F., \& Boulanger, F. 1990, A\&A, 233, 357

Chi, S., \& Park, Y.-S. 2006, J. Korean Astron. Soc., 39, 9

Combes, F., Encrenaz, P. J., Lucas, R., \& Weliachew, L. 1977, A\&A, 55, 311

Combes, F., Prugniel, P., Rampazzo, R., \& Sulentic, J. W. 1994, A\&A, 281, 725 de Vaucouleurs, G., de Vaucouleurs, A., Corwin, H. G., Jr., et al. 1991, S\&T, 82,

621, The Third Reference Catalogue of Bright Galaxies (New York: Springer) Draine, B. T. 1978, ApJS, 36, 595

Dufour, R.J. 1984, Structure and evolution of the Magellanic Clouds, IAUS 108, ed. S. van den Bergh, \& K. S. de Boer, 353

Eckart, A., Downes, D., Genzel, R., et al. 1990, ApJ, 348, 434

Kaufman, M., Bash, F. N., Hine, B., et al. 1989, ApJ, 345, 674

Kaufman, M. J., Wolfire, M. G., Hollenbach, D. J., \& Luhman, M. L. 1999, ApJ, 527,795

Knapen, J. H., Allen, R. J., Heaton, H. I., Kuno, N., \& Nakai, N. 2006, A\&A, 455,897

Kong, X., Zhou, X., Chen, J., et al. 2000, AJ, 119, 2745

Kramer, C., Stutzki, J., Rohrig, R., \& Corneliussen, U. 1998, A\&A, 329, 249

Garcia-Burillo, S., Guelin, M., \& Cernicharo, J. 1993, A\&A, 274, 123

Helfer, T. T., Thornley, M. D., Regan, M. W., et al. 2003, ApJS, 145, 259

Ho, L. C., Filippenko, A. V., \& Sargent, W. L. W. 1996, ApJ, 462, 183

Israel, F. P., \& Baas, F. 1999, A\&A, 351, 10

Larson, R. B. 1981, MNRAS, 194, 809

Loiseau, N., Nakai, N., Sofue, Y., et al. 1990, A\&A, 228, 331

Maloney, P., \& Black, J. H. 1988, ApJ, 325, 389

Nakai, N., \& Kuno, N. 1995, PASJ, 47, 761

Pellegrini, S., Cappi, M., Bassani, L., et al. 2000, A\&A, 353, 447

Rand, R. J. 1995, AJ, 109, 2444

Rand, R. J., \& Kulkarni, S. R. 1990, ApJ, 349, L43

Regan, M. W., Thornley, M. D., Helfer, T. T., et al. 2001, ApJ, 561, 218

Reuter, H.-P., Krause, M., Wielebinski, R., \& Lesch, H. 1991, A\&A, 248, 12

Rosolowsky, E., \& Blitz, L. 2005, ApJ, 623, 826

Rubio, M., Lequeux, J., \& Boulanger, F. 1993, A\&A, 271, 9

Sage, L. J., \& Westpfahl, D. J. 1991, A\&A, 242, 371

Sanders, D. B., Solomon, P. M., \& Scoville, N. Z. 1984, ApJ, 276, 182

Sakamoto, K., Okumura, S. K., Ishizuki, S., \& Scoville, N. Z. 1999, ApJS, 124, 403

Sakamoto, K., Fukuda, H., Wada, K., \& Habe, A. 2001, AJ, 122, 1319

Schuster, K.-F., Boucher, C., Brunswig, W., et al. 2004, A\&A, 423, 1171

Solomon, P. M., \& de Zafra, R. 1975, ApJ, 199, L79

Solomon, P. M., Rivolo, A. R., Barrett, J., \& Yahil, A. 1987, ApJ, 319, 730

Strong, A. W., Bloemen, J. B. G. M., Dame, T. M., et al. 1988, A\&A, 207, 1

Stutzki, J., \& Guesten, R. 1990, ApJ, 356, 513

Taylor, C. L., \& Wilson, C. D. 1998, ApJ, 494, 581

Taylor, C. L., Kobulnicky, H. A., \& Skillman, E. D. 1998, AJ, 116, 2746

Tenjes, P. 1994, Baltic Astronomy, 3, 180

Visser, H. C. D. 1980 , A\&A, 88, 159

Wild, W. 1999, The $30 \mathrm{~m}$ Manual-IRAM, Granada

Williams, J. P., de Geus, E. J., \& Blitz, L. 1994, ApJ, 428, 693

Wilson, C. D. 1995, ApJ, 448, L97

Wilson, C. D., \& Scoville, N. 1990, ApJ, 363, 435

Wilson, C. D., \& Scoville, N. 1992, ApJ, 385, 512

Young, J. S., \& Scoville, N. 1982, ApJ, 260, L41

Young, J. S., \& Scoville, N. Z. 1984, ApJ, 287, 153

Young, J. S., Xie, S., Taccondi, L., et al. 1995, ApJS, 98, 219 